\title{
Collective performance: modeling the interaction of habit-based actions
}

\author{
Michael D. Cohen*, Daniel A. Levinthal*** and Massimo Warglien ${ }^{\dagger}$
}

Recurring patterns of action are essential in our efforts to explain central properties of business firms and other organizations. However, the development of systematic theory has been hampered by the difficulty of adequately specifying foundational assumptions. We address this problem by defining a concept of collective performance, which brings together a range of recurring organizational action patterns that have been studied under labels such as "routine," "practice," standard operating procedure, or "genre of action." All these forms of organizational action are based on human habit to a significant degree. We propose a conceptual framework for such habit-based organizational action patterns. The framework is a set of core principles and desirable model properties that can serve as a guide in the development of formal models of collective performance. It provides micro-foundations for the modeling of collective performance that are aligned with contemporary developments in psychology. Finally, we present a series of examples, developed in Supplementary Materials, that shows how our framework leads to new classes of formal models that can aid the analysis of collective performance.

JEL codes: D21, D7, M10.

\section{Introduction}

Recurring patterns of action are a central feature of organization. Organizations get better at what they do by exploiting the similarities of past experience to the

${ }^{\star}$ Michael D. Cohen, School of Information, University of Michigan, Ann Arbor, MI 48109, USA. e-mail: mdc@umich.edu

${ }^{* *}$ Daniel A. Levinthal, Wharton School, University of Pennsylvania, Philadelphia, PA 19104, USA. e-mail: dlev@wharton.upenn.edu

†Massimo Warglien, Università Ca Foscari de Venezia, 30123 Dorsoduro Venezia, Italy. e-mail: warglien@unive.it

"Main author for correspondence. 
requirements of current tasks. If this were not commonplace, organizing would confer far less advantage than it does, and would be a far less pervasive feature of social life. Accordingly, many social science theories rely on distinctive properties of recurring organizational action, such as capabilities theories in the field of strategy (Cyert and March, 1963; Nelson and Winter, 1982; Dosi et al., 2000; Teece et al., 1997), or theories of organizational culture (Weber, 2005), or of organizational change (Feldman, 2000).

It has become clear, however, that although the existence of such action patterns seems clear, research consensus about their properties has been hard to achieve (Felin and Foss, 2009; Cohen et al., 1996; Feldman and Pentland, 2003; Cohen, 2007).While many researchers have worked with the label "routine," others have preferred alternatives such as "practices," "standard operating procedures," "high reliability" action, or "genres of action" (Orlikowski, 2002; Cyert and March, 1963; Weick and Roberts, 1993; Yates and Orlikowski, 2002). Even though all these approaches focus on action, as contrasted with deliberative choice or problem-solving, there have been sharp debates about whether such recurring actions are "mindful" or "mindless" (Levinthal and Rerup, 2006; Weick and Sutcliffe, 2006; Weick et al., 2005). Recurring patterns of action have been seen as bulwarks of stability by some, or as building blocks of change by others (Ashforth and Fried, 1988; Feldman and Pentland, 2003). They receive credit for organizational efficiencies such as steeply climbing learning curves (Argote, 1999) and blame for avoidable calamities such as the Tenerife airport disaster (Weick, 1990) or wrong-site surgery (Seiden and Barach, 2006). To make sustained and shared intellectual progress, we clearly need closer agreement on the properties of these recurring organizational action patterns, which we will group together under the label collective performance.

There are many lines of work that may contribute to resolving these problems and that should be pursued including controlled experimental study, insightful ethnographic observation, simulation, and careful verbal theorizing (Kane et al., 2005; Howard-Grenville, 2005; Pentland et al., 2010; Rico et al., 2008). In the present article, we demonstrate the potential for developing formal theories that can illuminate the properties of collective performance. While formalization has its limits in the study of organization, it can foster clearer distinctions between contending positions and higher standards of mutual coherence among arguments. These properties are badly needed if we are to clarify the issues that have bedeviled the study of collective performance.

There have been relatively few efforts at formalization in this domain. ${ }^{1}$ One of the major barriers to productively formalizing recurring organizational action patterns

\footnotetext{
${ }^{1}$ Nelson and Winter (1982), Pentland and Reuter (1994), Abell et al. (2008), and Chassang (2010) are some leading examples, but these and other attempts have attracted relatively few followers. While the notion of routine-driven firm behavior in Nelson and Winter has had enormous influence, it is the general conceptual arguments (Chapters 4 and 5) and not the specific formal modeling apparatus that they offered that has been so widely embraced.
} 
has been the difficulty of representing several of their key features. We suggest that the various forms of collective performance all involve action that is substantially rooted in human habit, and that they therefore reflect processes of determining action that contemporary psychology has shown to be quite different from rational—or even boundedly rational—decision making.

As a result, calls for formalization of the "micro-foundations of routine," such as those made by Felin and Foss $(2005,2009)$, are, on the one hand, quite appropriate, but on the other hand seriously incomplete. We do indeed need greater precision and logical coherence, as has been argued, but the formalizations we employ must be consistent with the psychological processes of actors whose actions are determined in large part by learned habits and associations rather than by deliberating over the likely consequences of exogenously defined alternatives. For-as has long been recognized-it is these habit-based processes that give routines, institutionalized practices, or organizational customs their distinctive common qualities (Aristotle, 1992; James, 1981; Dewey, 1922; Giddens, 1984; Bourdieu, 1990).

We need simultaneously to achieve both more precise formalization and better fidelity to the underlying psychology of habit. ${ }^{2}$ A crucial building block is the representation of an action as a mathematical function. That commitment leads to a focus on how to represent the situation, its perception by the actors, and the ends that are currently activated. These elements provide the action function's inputs, triggers, and anticipated results.

Developing further this perspective, we propose a set of 12 desirable modeling properties and argue that formal models should conform to as many of them as possible. Taken together, the principles and properties constitute a framework for modeling collective performance.

In the main text-and in the Supplementary Materials-we provide illustrative model fragments and interpretations of prior research that give a clearer sense of the promising contributions better formal modeling can make to understanding collective performance. Models developed along the lines of this framework should provide

\footnotetext{
${ }^{2}$ The term "habit" has received a variety of definitions in the psychological literature. We follow the work, stretching back to Dewey and James, that has defined habit broadly, as in the authoritative review by (Graybiel, 2008). "First, habits (mannerisms, customs, rituals) are largely learned; in current terminology, they are acquired via experience-dependent plasticity. Second, habitual behaviors occur repeatedly over the course of days or years, and they can become remarkably fixed. Third, fully acquired habits are performed almost automatically, virtually non-consciously, allowing attention to be focused elsewhere. Fourth, habits tend to involve an ordered, structured action sequence that is prone to being elicited by a particular context or stimulus. And finally, habits can comprise cognitive expressions of routine (habits of thought) as well as motor expressions of routine." This approach differs from a narrower definition that limits the scope of habit to actions that have become completely independent of goals or motives. While advantageous for laboratory work (Wood and Neal, 2007), this scope is too restricted for our field-relevant intentions. (On this question also see Seger and Spiering, 2011; Cohen, 2012.)
} 
rich possibilities for clarifying current theoretical tangles and for illuminating the central role of collective performance in the study of organization.

\section{Conceptual overview}

Our label "collective performance" gathers together many forms of recurring organizational action that are distinct from choice or decision-making, and in which habit plays a major part. Therefore, the approach to formalizing action plays a vital role in our framework. One of our central commitments is to represent an action pattern as a mathematical function, a generalized procedure that transforms an input into an output. ${ }^{3}$ Functions are defined over domains, the sets of inputs they can operate on. ${ }^{4}$ What is central in our approach is that functions apply to novel input values, to situations they have "never been seen before." This allows a function to correspond to an action pattern, or to a part of one, while instances of applying that function to a concrete situation, which we call acts, can be quite distinct-indeed unique. This representation of action patterns and acts provides the basic structure for addressing the fundamental issues of pattern, recurrence, and categorization that have vexed theorizing about recurring organizational action patterns (Weick, 1995; Birnholtz et al., 2007; Pentland and Feldman, 2005).

Many consequences flow from this fundamental commitment. As noted, a function that models an action pattern must operate on some input. We label that input a situation, with the idea that it may represent not only the actual and perceived condition of the actor's material environment, including tools and the state of work in progress, but also the other agents involved in a collective performance. Each significant variation defines a distinct situation, and actors may divide situations into categories. The function that represents an action pattern potentially applies to a set of situations that fall within some category.

This approach will allows us to explore category structures that are not just fixed entities but that may adapt to individual and collective experience. As we show in a set of models in the Supplementary Materials, certain attributes of a setting may

\footnotetext{
${ }^{3}$ It is important to note that by postulating a function to represent action, we are not implying that a given action will result in a unique, deterministic outcome. Further, the existence of some (possibly one to many) mapping from act to outcome does not imply that this mapping is well understood and known to the actors as is the convention in the context of a production function in neoclassical economics.

${ }^{4}$ A familiar example might be $y=x^{2}+3 x+7$, defined for a domain such as the values of $x$ that are odd integers. For any $\mathrm{x}$ that is in this domain, the function will transform it into a new value, $\mathrm{y}$. While this particular example is homely, the notion of function is impressively abstract and powerful. Functions can be arbitrarily complex, and even nonnumerical in their inputs and outputs. They can even be defined independently of their domain, for example, via lambda calculus (Barendreght 1984).
} 
become more or less salient and thereby shift a particular situation's category membership. Categories can also undergo significant degrees of mutual adaptation, a critical process in the emergence of stable action patterns among individuals.

Acts transform definite elements in a given category, producing new situations that may themselves be expected or desirable-or may be unexpected, or undesirable. Thus our approach to collective performance explicitly takes into account the expectations and activated-ends that may interact with the action pattern. Of course, the new situations resulting from a given act may themselves prompt the actions of others. Thus, a patterned collective performance may be represented as an ensemble of categorized situations, action patterns, and desirable ends. These three major elements are woven together to form the basic line of our argument. We shall see that each has its own detailed structure and important dynamics. We believe that much progress can be made through thoughtfully combining formal representations of situation perception, of action as a function, and of ends that can be activated as well as being exogenous initiators.

Accounts of collective performance in realistic circumstances will also have to accommodate instances in which patterned behavior "breaks down." Such events are key moments in processes of organizational change. A breakdown might arise, for example, when another's act yields a situation that falls outside an actor's existing category structure, so that no continuing action seems appropriate, or when an act yields an outcome that is not satisfactory. Breakdowns may require actors to create new representations of their local environment, to consider anew what situations are prototypical and what critical dimensions distinguish one setting from another, or to revise their notions of what is an acceptable or desirable end to be realized by action. They can drive the dynamics of collective performance systems, and may sometimes be moments of transition from habit-based action to reflective analysis and choice.

\subsection{Definitions and properties: a framework for modeling collective performance}

In this section we lay out the principal elements of our framework more carefully, marking the first occurrence of key terms with bold italics. As a terminological stipulation, we will use habit or skill for a recurring action pattern of an individual, and collective performance for an action pattern of a group. As we noted earlier, we use the phrase "collective performance" for actions that are substantially based in habit and take it to include, but not be limited to, action patterns that have conventionally been designated with "routine," "standard operating procedure," "genres of action," or "organizational practice." In our approach, collective performances may reflect wide ranges of processes for determining desirable ends, flexibility of action possibilities, and categorizations of situations. We hope that the new term will escape distracting connotations of mindlessness and rigidity called up by the word "routine," connotations which have plagued efforts to think carefully about recurring organizational action patterns (Birnholtz et al., 2007; Cohen, 2007). We also hope 
this set of key terms will keep clear the distinction between individual and collective levels of analysis, a distinction that can be muddled with the terms such as "routine" and "practice" which are frequently used at both levels.

We follow Cohen and Bacdayan (1994) and Cohen (2012) in taking the recurring action patterns of group members to be remembered largely as individual habits. More precisely, these are action patterns that are retained substantially through the form of human memory that stores habits and skills and is often designated as "procedural" or "non-declarative" - in contrast with "declarative"-memory (Squire and Kandel, 1999) and that rely on what has been termed "dorsal" perceptual apparatus (Norman, 2002). In addition, we presume that habitual activities of individuals and collective performances are, in general, not mindless. If we need to indicate that pathological extreme in which activity is repeated without due regard of context, we will use the expressions "dead habit" or "dead routine" (Levinthal and Rerup, 2006; Cohen, 2007). ${ }^{5}$

In our view, models of collective performance should be more extensively grounded in sound contemporary psychology. We believe that our framework will make it easier for subsequent work to draw on the strong developments in the study of individual perception, action, and thought now occurring in psychology (Jeannerod, 1997; Gärdenfors, 2000; Norman, 2002; Sebanz et al., 2006; Rizzolatti and Sinigaglia, 2008; Ping et al., 2009; Massen and Prinz, 2009; Bargh et al., 2010; Neal et al., 2012).

Our starting point is the assumption that a productive model of collective performance must take into account the habit-based actions of the participating individuals, the ends they have in view, and their perceptions of their situation. In accordance with this principle, we develop a set of desirable model properties within our framework. We present them in three sections:

- Properties regarding representation of perception,

- A property regarding desirable ends, and

- Properties regarding representation of action, the relations between perception and action, and the relations between actions.

The proposed properties, which are summarized for convenience in Table 1, are hardly radical. We expect many readers, considering each one separately, might think them nearly obvious. But our position is that they form a coherent package, and that models that simultaneously satisfy many or all of them have the best chance of clarifying the workings of habit and collective performance.

\subsubsection{Properties regarding modeling of situations}

Since we believe that the habit-based action that constitutes collective performance is triggered by perceived situations, we take it as fundamental that a model should

\footnotetext{
${ }^{5}$ Paul Ricoeur (1975) develops the same idea in his book on La Métaphore Vive. An argument for different terminology is in Weick and Sutcliffe (2006).
} 
Table 1 Summary of framework properties

Property Property description

label

Representation of perception

A Model a highly differentiated perception of the world, including the observed or expected actions of others

B Model relations of similarity among situations

C Allow for dynamic change in the dimensions or properties ascribed to situations, as a result of experience

D Reflect the actor's categorization of possible worlds

\section{Desirable ends}

E Encode activated-ends, classes of situations that are desirable for the actor in the context of perceptions and other actions take by the self and by others.

Representation of action, the relations between perception and action, and the relations between actions

F Model a specific act, the event of an actor transforming a definite current situation (a point in a situation-set) into a new one

$\mathrm{G} \quad$ Model an action as a function, or a collection or composition of functions

$\mathrm{H} \quad$ Model the composition of acts, or actions employing several smaller ones to accomplish something larger

I Represent the compilation of elementary actions into still larger actions that can themselves be treated as units.

J Model feedback from the outcomes of specific acts that might dynamically alter the structure of situation and action spaces.

K Model dynamic processes of short-run development of situation perception, action to be taken, or ends to be pursued, to allow for inter-activation among them

$\mathrm{L}$ Represent processes that produce alignment or coherence among the actions undertaken by the multiple individuals participating in a collective performance

represent in some way each actor's perception of the possible worlds in which she might find herself. There are many ways this might be done, each with its own modeling consequences. We make specific choices in our illustrative examples below and in the Supplementary Materials, but here we want to specify the properties at a more general level. ${ }^{6}$ We refer to such a representation generically

\footnotetext{
${ }^{6}$ We provide an alphabetical label for each property so that we may reference them throughout the remainder of the article.
} 
as a situation-set. A model that includes a situation-set should have a number of key features:

(A) It should be able to depict a fine-grained encoding of the actor's perception of her current situation, including the observed or expected actions of others.

(B) It should support modeling of relations of similarity among situations.

(C) It should allow for dynamic change in the dimensions or properties ascribed to situations, as a result of experience.

(D) It should be able to reflect the actor's categorization of those possible worlds.

\subsubsection{A property regarding desirable ends}

Collective performances are not aimless. They are important precisely because they so often play an instrumental role in organizational life, transforming the current world into a new one. These actions are typically guided by a representation of desired results.

(E) It should be able to encode activated-ends, classes of situations that are desirable for the actor in the context of perceptions of situations and of other actions taken by the self and by others.

Our approach certainly allows for the conventional portrayal of exogenously specified goals or intentionally selected objectives, but may also encompass cases where there is strong priming of ends by similar prior experience, by the presence of other actors or tools, by immediately prior actions, or other perceived aspects of the action context (D'Adderio, 2011). (See K. below.) This flexibility is essential for an adequate representation of action strongly grounded in habit.

\subsubsection{Properties regarding modeling of action, of relations between perception and} action, and of the relations between actions

Since we take habits and collective performance as recurring patterns of action at the individual and organizational levels, a model should be able to represent in some way an actor's possible and actual transformations of her current situation into a new one by interacting with her world and other actors. Again, there are many ways this might be done, each with distinct modeling consequences. Whatever the approach, a model of action patterns should have several key features:

(F) It should be able to model a specific act, the event of an actor transforming a definite current situation (a point in a situation-set) into a new one by a specific interaction with her environment.

(G) It should model an action as a function, or a collection or composition of functions, identifying the regions of a situation-set in which the action is believed possible, associating the definition with the actor who carries out the action (whether self, another, or some category of others) and the expected region of the situation-set that should result. 
(H) It should be able to model the composition of acts, or of actions, employing several smaller ones to accomplish something larger, such as two acts carried out simultaneously or in sequence.

(I) It should be able to represent the compilation of elementary actions, fusing them into still larger actions that can themselves be treated as units.

The pair of properties $\mathrm{H}$ and I allows for a distinction between deliberate assemblages of distinct acts (or actions), such as purchasing milk, then calling one's spouse to avoid a duplicate purchase, and the fusion of repeated groups of acts (or actions) into larger units, typically by means of repetition (e.g., open garage door-enter car-insert key—start car-back out of garage).

(J) It should be able to model feedback from the outcomes of specific acts that might dynamically alter the structure of situation and action spaces.

(K) It should model the short-run dynamics of situation perception, action to be taken, or ends to be pursued, to allow for inter-activation among them.

That is, it should be possible for potential action to induce changes in situation perception in which ends are activated (as detailed in E. above). This is an important broadening of typical assumptions that take ends as fixed initiators of perception and resulting action. Substantial bodies of psychological evidence have accumulated to indicate that this extension is necessary to capture characteristic dynamics of action strongly based in habit (Aarts and Dijksterhuis, 2000; Bargh, 2005; Morewedge and Kahneman, 2010).

In order to accomplish a collective performance, a recurring pattern in which the actions of multiple actors are engaged, a model must also represent one or more principles of mutual coherence that govern the relations between actions of the participants. This leads to a further property:

(L) It should be able to model processes that produce alignment or coherence among the actions undertaken by the multiple individuals participating in a collective performance.

While noted as a single property, the emergence of alignment and coherence among a set of individuals is central to our overall argumentation and merits substantial elaboration. Notions of "coherence" are fundamental to traditional economic approaches to interactive behavior where the characterization of "equilibrium" is the core of any analysis, whether modeling the strategic interaction among a dyad as typical in game theory treatments or broad economic collectives as in rational expectations models. Of course, as demonstrated by work on evolutionary game theory, one can have enduring, stable behavioral patterns even in the absence of forethought or intention.

The issue of mutual compatibility of actions in the context of collective performance is not defined in terms of the persistence of behaviors, but rather is a form of an 
interactive bounded rationality, where coherence is a joint consequence of how situations and outcomes are encoded and the acts associated with these encodings. The joint categorization of contexts, behaviors, and resultant end-states by this categorization of contexts is coherent in the absence of a breakdown, where a breakdown may stem from either the failure to achieve an activated goal on the part of one or more parties or for an act to generate a state outside the category schema of one or more actor.

There are many psychological processes that can be templates for model elements that generate this property of multi-actor alignment (Sebanz et al., 2006). Indeed, an important promise of a more detailed and psychologically realistic portrayal of individual actors is its potential to clarify and distinguish the multiple possible mechanisms of assembling collective performance. The properties A-K ascribed to individuals offer potential mechanisms for the increase of coherence among interacting individuals. For example, one person's action can activate ends in another (E); situation perception can become productively realigned through interaction among participants (C); and compilation can occur across actions of multiple actors (I). We consider the issue of the alignment of categorization and action below in Section 3.2. We discuss the role of artifacts and boundary objects as providing common anchors (Star and Griesemer, 1989) and affordances (Gibson, 1979) in facilitating the alignment but, in addition, in the Supplementary Materials we develop a model of associative learning and mutual adjustment of category structures consistent with the micro mechanisms specified above.

\subsection{Elaboration of the main properties}

We now discuss the considerations that have led us to suggest these principles as useful guidance to modeling work in the area of habit and collective performance. In the sections after this one, we present more formal illustrations of the general approach we have outlined.

It is important to recognize these properties not merely as independent commitments, but as interdependent elements of a coherent system. Because of their high degree of interdependence, we present the discussion in a more discursive format, referring back to the main principles by their corresponding letters. We also draw on the context of automobile assembly for a set of running illustrations of the principles and properties under discussion, moving back and forth between realistic details and the requirements models must meet to reflect those features.

Auto assembly provides an iconic example of a production routine. It has often been stereotyped as the sort of mindlessly executed behavior we have termed "dead routine." But it clearly can involve much more flexible form of collective performance, particularly as exemplified by the continuous innovation and refinement found in approaches such as the Toyota Production System, which will be the source of a number of our illustrations. 
We believe that it is important that a model of collective performance should represent a situation-set that accommodates a high-and dynamic-degree of differentiation (A, C). Vast amounts of experience and research show that habits of individuals and collective performances of groups are finely conditioned by the circumstances they perceive themselves to be encountering (Dewey, 1922; Pentland and Rueter, 1994; Feldman, 2000; Polanyi, 1958). Thus, it is critical that a persuasive model of habit-based action allows for the conditioning of an act upon the actor's perception of the current conditions (D, F). Much of the effectiveness of assemblyline routine for automobile manufacturing can be traced to the rich visual inputs from partially completed work and the actions of coworkers that entrain the actions of participants (I, L). Quality improvement approaches, such as the Toyota Production System (Liker, 2003), add to these well-defined category structures that indicate situations of conformance or nonconformance to performance standards (J). Indeed, Adler and Cole (1993: 89) suggest that "without a well-documented, standardized process, it is hard to imagine how these people could have spotted improvement opportunities."

As Arrow (1972) argued, a critical role of organization, and more generally the facilitation of coordinated behavior, is to help foster a common code or language among actors. This language can encode shared categories $(A, B, D)$ that facilitate coordination and joint problem solving $(\mathrm{L})$. We see this strongly expressed in the context of the Toyota Production System in the development of general heuristics of joint problem solving (Adler, 1993; Liker, 2004). MacDuffie (1997) observes a quite refined, context-specific language in his examination of the production process on the paint line. The Quality Analysts for the paint department refers to defects as "mutilations" and had rich nomenclature for the differing forms of mutilations: boils, craters, bulls-eyes, sags, runs, orange peel, dings, mars, scratches, cracks, grind marks, and powder bumps. It is important to note that these terms were not idiosyncratic to a specific quality analyst, but that considerable effort had been made to ensure that all the relevant actors used the same categorial lexicon for describing possible deficiencies in the paint. Indeed, this effort extended beyond the shop floor to include the dealers as well when filling out warranty filings.

The set of actions available to a typical person is truly enormous. We can begin with the tens of thousands of words that an adult can correctly use in context, and go on to the many more life- and work-skills that are also commonly mastered. It is reasonable to suppose that these number at least in the hundreds of thousands. For the most part, these capabilities are exercised not in random circumstances, but rather only in circumstances to which they are-with occasional embarrassing exception-highly appropriate. This wide range of possible actions is acquired over a lifetime and is augmented through experience and explicit training in educational and work environments (F, G, H, I). If models are to provide insight into how collective performances change as a result of experience, the representation used for a situation-set-and for categorization within it-will need to accommodate 
the occurrence of such learning (C, J). Adler and Cole (1993) refer to the Toyota production systems as democratic Taylorism. At any point in time, action patterns are highly scripted, but the possibility of an enormous variety of potential actions is presumed, both in the perspective of individual workers who are encouraged to entertain alternative acts and of a broader organizational system receptive to such initiatives.

Because life is filled with circumstances that are not perfectly identical to any that have been previously encountered, a good model of habit-based action should make it possible to represent categories within situation-sets (D), so that situations that are not identical can nonetheless be treated as instances of larger established patterns. If you are driving home at the end of the day, the cars and conditions on the road are never perfectly identical to a previous trip, but the situation is usually still an instance of a well-established category.

A closely related requirement is that a conceptualization of a situation-set should support measures of similarity among situations (B). Action may be called for in situations that are not already members of preexisting categories. A model incorporating a concept of similarity can accommodate the searching out of situations that share important features with current novel conditions and that suggest possible actions (J). It also allows for the formation of new categories from situations deemed similar (B, D). Many different notions of similarity are available from work in psychology and philosophy. MacDuffie's (1997) contrasting study of problem solving at three auto plants identifies different modes of categorization that had profound impact on the problem-solving process. Two of the plants (GM and Ford) had a strict categorization of problems; Ford, for instance, encoded problems as being one of design, vendor, or plant. As a consequence, design engineers only engaged with problems encoded as design problem and ignored problems associated with the production process, leaving these to the plant engineers, even if often problems involved a mix of design and production issues. In contrast, MacDuffie (1997) found that at Honda there was an emphasis on seeing problems in their context, in situ, and problems were placed in "fuzzy" categories defined by prototypical examples. MacDuffie finds that this approach allowed for a richer and more informative category structure and clearer communication from one organizational member to another.

\section{Acts and Actions}

The world is transformed when an actor interacts with it through a specific act. We are sharply distinguishing such concrete acts from the actions, modeled as functions, of which the acts are instances $(G, F)$. Incorporating functions into the modeling of action makes possible a much clearer discussion of what is the same and what is different about any particular acts by referring to the functions involved and their domains. 
The new situation resulting from a specific act is also perceived by the actors (A, C, J), and from it a further act could be triggered. So, sequences or ensembles of acts can unfold. We refer to the sequencing or assembly of acts—or of actions-as "composition," and actors commonly do this either in response to an evocative situation or deliberately in pursuit of a goal or objective (H, E). This "composition" need not be restricted to a focal individual's own set of actions. Importantly, it may include the actions of others as well.

Achieving activated-ends, or failing to do so, can trigger adjustments of other elements in a model structure. The scope-or even existence-of categories of actions or situations might change as a result $(\mathrm{D}, \mathrm{J}, \mathrm{K})$.

A possible collective performance will normally be a composition of actions, while its realization will normally be a composition of specific acts. We can begin to see that this simple distinction $(F, G)$, together with fundamental roles for similarity and category (B, D), allows the development of models that can resolve confusions over the "sameness" of habit-based action patterns. Acts will generally be distinct in their details, each arising in and conforming to a unique situation. But acts from common categories of action may be perceived as indistinct, as instances of "the same" action patterns (Birnholtz et al., 2007).

Observation shows that there is a form of relation among actions that is more strongly integrated than simple composition of separate actions. To illustrate this at the level of individual habits, the elementary movements of individual muscles can become fused into recognizably larger units such as grasping or stepping. Those units can become tightly integrated in turn into still larger units such as eating or walking. These larger ensembles are so well integrated that we comfortably treat them as action patterns in and of themselves. Indeed, the low level components can even be replaced, albeit with effortful attention, as when an injured person receives an artificial leg and learns to walk again.

We refer to this process of tightly integrated composition as compilation (I). ${ }^{7}$ The idea of compilation suggests how models might represent the accumulation of larger scale action patterns, habits, or collective performances that might be effective, but might exhibit some inertia, or resistance to change.

While both scholars and practicing managers use the label "Toyota Production System" to reference a broad organizational capability and a general set of practices, the constituent team members can often be seen performing work processes at a

\footnotetext{
${ }^{7}$ In choosing this term, we are following a suggestion of the psychologist John Anderson (Singley and Anderson, 1989) that the accumulation of larger skills resembles the formation for computing devices of large executable blocks of low-level machine instructions. The analogy is not exact, however. As we have observed, unlike compiled computer instructions, with sufficient time and thought, aggregate units of action such as habits of eating or walking, can sometimes be decomposed into meaningful sub-actions and reconstructed.
} 
vastly more micro level. Adler and Cole (1993) observe the composition and aggregation of initially distinct acts rapidly become recurring 60-second actions, an extraordinary example of compilation. This contrast is further highlighted by the scaling up process in the context of a new product on the production line. When beginning with a new model, workers continue to act in 60-second units; however, workers took time in between each 60-second cycle to consider problems and opportunities for improvement that may present themselves, yet, "within the first week, the pace quickened from one car an hour to one car every 10 minutes" (Adler et al., 1999: 58). After regaining familiarity with the production process, the production of an automobile once more became a compiled action, rather than a series of distinct, identified acts.

Using notions such as activated-ends to delineate an aimed-for region of the situation-set (E), and of compositions of actions $(\mathrm{H})$, the properties provide a strong basis for models in which collective performances may not unfold as expected. An act, being an interaction of a perceiving actor with a partially independent world $(\mathrm{A}, \mathrm{F})$, and with the actions of other actors (L) may fail to reach a region of the situation-set from which a typical next action may be smoothly undertaken. The end of a sequence of acts may be a point in the situation-set lying outside the region of activated-ends. These conditions correspond well to observations of "breakdowns" in the empirical literature, circumstances in which the expected result has not been reached, or the expected continuation of action is not feasible, or not satisfactory. These are circumstances that could trigger activities of "repair," selecting of new acts that are plausible and/or changing the category structure of situations or actions. Such repairs may reconstruct the system of activity to better deal with its evolving world (C, J, K). Indeed, the lean production system of Toyota (Adler and Cole, 1993; Cusumano, 1985; Womack et al., 1990) was designed in part to prompt "breakdowns" in order to highlight inefficiencies and provide a focus for possible efforts at improvement. A strength of the framework articulated above is that it can encompas a broad range of collective peformances, ranging from highly compilled action patterns that might even approach the sterotype of "dead routine," through the more fluid actions patterns of flexibly reoccuring organizational actions, whether University move-in routines (Feldman, 2000) or reenactment of summer camps (Birnholtz et al., 2007), and on to the even more dynamic patterns of a jazz performance (Barrett, 1998; Berliner, 1994; Weick, 1998).

Thus, these basic properties define habit-based collective action as being a distinct form of action, differing not only from rational choice conceptions, but also from classic images of bounded rationality. Further, while laying out a distinct domain, the properties are also able to encompass a wide range of behavioral patterns.

In the following section, we begin to lay out more specific illustrations of these general modeling properties, followed by the characterization of one extended illustrative model. 


\section{Situations, actions, and performance: illustrating the modeling framework}

The basic commitments of our general framework for modeling collective performance are perceived situations, actions represented as functions, and activated-ends. These crucial architectural principles imply our list of properties, detailing how models can best represent their structure and interactions.

While the specific modeling assumptions that are made to instantiate the components of the modeling framework are clearly critical and must be adapted to a researcher's immediate purposes, the general conceptual structure developed here helps frame such particular instantiations. We provide a series of illustrative framework elements in this section and point to further examples in the Supplementary Materials.

\subsection{Situation-sets}

Our framework posits that there are sets of situations, where situations are representations of states of the world. However, we need not presume that these situations necessarily correspond one-to-one with actual states of the world, and indeed, as a limiting case they might be entirely decoupled from the actual setting. Situation-sets in models may have different structures. They may be simply collections of situations or be endowed with some richer structure, e.g., of a geometric or topological kind (Gärdenfors, 2000) or of a logical one (Barwise and Perry, 1983). ${ }^{8}$

In general, the framework suggests that situation-sets can be decomposed into situation categories, through the workings of some kind of perceptual or conceptual categorization. There are many possible ways to model the structure of a situationset. For example, categories are often organized around prototypical situations (see Figure 1 for an illustration and see [Rosch and Mervis, 1975]). If the situation-set is a space with some geometric structure (i.e., endowed with a similarity metric), one can define categorization as a decomposition of the space into convex subsets (Gärdenfors, 2000; Warglien and Gärdenfors, 2013) where each convex subset is determined by the similarity of a situation to the closest prototype. (This is the strategy we adopt in model 1 in the Supplementary Materials). Other approaches to categorization have been proposed in the recent economics literature (Mullainathan et al., 2008).

\subsection{Actions and activated-ends}

In our initial presentation of the framework, we suggested that actions be defined as functions. We can now be more precise in illustrating this approach. The basic idea is

\footnotetext{
${ }^{8}$ We use the term situations in our framework in its broader common language usage, not in the technical usage of the (Barwise and Perry, 1983) formal logic, "Situation Theory."
} 


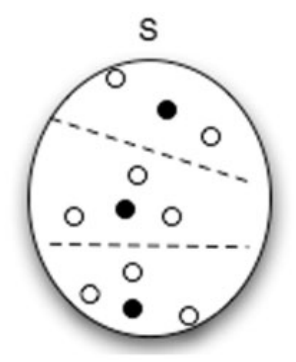

Figure 1 A situation-set. (Dashed lines separate categories of situations. Black dots are category prototypes).

that actions should be represented as "generalized procedures" transforming input situations into output situations. However, some care is needed to represent the subtle structure of actions. We proceed from the representations of acts, a simpler entity than actions (see discussions of properties $F$ and $G$ above). With the idea of a situation-set, we can represent an act as a transformation of a specific "input" situation into an "output" situation, a (typically new) point in the situation-set."

Acts can be chained to generate a composed act as illustrated in the top panel of Figure 2.ii. The composed act can be compiled (Singley and Anderson, 1989), creating a larger unit and suppressing the prominence of the original components, as illustrated in the bottom panel of Figure 2.ii.

Modeling may sometimes require a concept of an action that is more general than this simple one. In what follows, we show how action may be modeled as a tunable family of partial functions (Davey and Priestley, 2002). Our explanation of this requires a few steps.

An act is a transformation of a single situation into a new one. However, what we call an action usually applies to many situations. For instance, we can push objects that are in many different locations. It will often be natural to express this with the notion of a function that carries each point in the set of input situations into a corresponding output point. However, it can sometimes be important to recognize that an actor's representation may not map all input situations into output situations. There could be objects in locations where pushing has never been considered. In general, an action will map a subset of situations (typically a situation category) into another one. This corresponds to the concept of partial functions, each having as a domain of application a subset of the situation-set (Davey and Priestley 2002). Using partial functions conforms to the fact that humans may not recognize all the situations to which an action might apply_the function will be undefined on such

\footnotetext{
${ }^{9}$ We note that the framework can accommodate probabilistic mappings to sets of points in the situation-set, along the lines of the notion of an act in standard decision theory (e.g., Savage, 1954). Here we use the deterministic formulation for ease of exposition.
} 


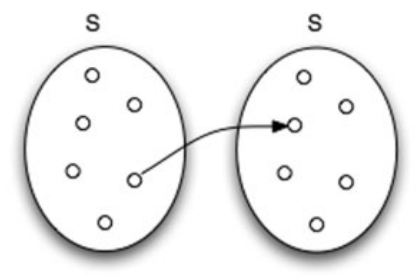

(i)

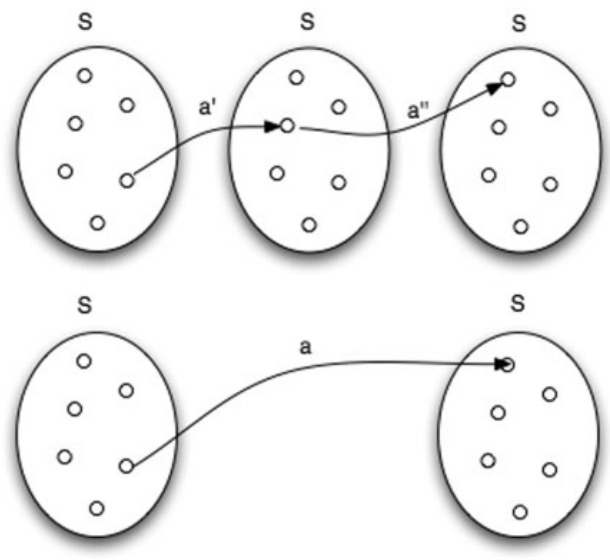

(ii)

Figure 2 (i) An act. (ii) Composition and compilation of acts.

inputs. This provides some plasticity, in that the set of situations to which an action may be applied may change over time, e.g., due to changes in categorization of situations. An example of this is provided in model 3 in the Supplementary Materials.

In addition to applying to many situations, an action may also produce many subtle variations in its expression, even if the starting point of activity is virtually identical. An object may be at the same initial location, but we might push on it with more or less force. The new situation that results thus could be different, with the object moving a greater or lesser distance. Usually small differences in force will produce small changes in distance, but there could be threshold effects producing no motion (the object is stuck), or extreme motion (the object falls over). So an action can be conceived of as a family of similar partial functions, related by parameters that capture ("tune") the variability in how an action could be carried out from a given input situation-all the levels of force that might be applied in pushing the object from its given location.

In general, these families of functions will not be arbitrary, but related by structural similarity. For example, "crossing a park" might express a family of trajectories that are continuously deformable into one another, and that are characterized by some topological constraints (e.g., the trajectories cannot pass outside the park...). This corresponds to the linguistic fact that lexical items used to express actions refer to bundles of similar transformations, rather than to specific ones (Warglien, Gárdenfors and Westera, 2012).

This definition of action as a tunable (parameterized) family of similar partial functions will allow us to give a richer account of an action, one that represents not only the transformation that is produced but also the manner in which it is 
accomplished. It corresponds to the finding of linguists that verbs can be divided into two categories reflecting two complementary aspects of an event: the result to be accomplished ("relocate the object") and the manner of accomplishment ("nudge the object") (Levin and Rappaport Hovav, 1991). The abstract form of this relationship is shown in Figure 3.

Just like acts, actions can be composed and compiled, as shown in Figure 4. Since they consist of partial functions, however, their composition is not to be taken for granted: one may discover in "composing" one action with another that the domain of the second matches the range of the first. But if this is not true, an intended composition of actions may be locally undefined, corresponding to one form of our concept of breakdown.

With the aid of this concept of action, we can build up the subsequent stages of our notion of collective performance. The concept of action illustrated so far is not sufficient. It represents the manner of accomplishment, in the sense of the tuning of activity to a specific situation, but not the determination of the actions that will constitute the performance-the selection of which specific function will be applied to a specific situation. ${ }^{10}$ Performance in our framework is sensitive not only to the properties of a given action, but also to the situation, the activated-ends, and the repertoire of possible combinations of actions that might accomplish those ends.

As we noted above, we use "activated-ends" to indicate a subset of the situationset toward which the current situation is to be transformed. The subset could be determined by an intentional choice to achieve certain conditions, or by psychological processes like situational priming, or by some combination of the two. While in principle the activated-ends subset might be determined by either process, the characterization of subsequent actions may be substantially different.

The actions that constitute a performance are also determined by a structure we label the repertoire, a collection that classifies manners of accomplishment according to the results to be accomplished. So, for example, the alternative manners of moving a heavy object might include pushing it, pulling it, or even rolling it, should it happen to be round. Once more, we do not intend to incorporate intentionality directly into this concept. The knowledge in the repertoire changes over time as a consequence of the actor's experience and the contents of a repertoire may be applied to many different action situations for the accomplishment of many different ends.

Now we can assemble the elements of our framework for a collective performance. Introducing a repertoire allows us to conceive a performance as the (partial) mapping of the product of the repertoire and the situation space into the situation space itself.

Consider as an example an agent who is about to perform a common activity, such as rearranging the tables for the upcoming lunchtime in the restaurant where he

\footnotetext{
${ }^{10}$ Mathematically speaking, this refers to locating in a space of functions a point corresponding to a function that is appropriate to the situation (Lawvere and Schanuel, 1991).
} 


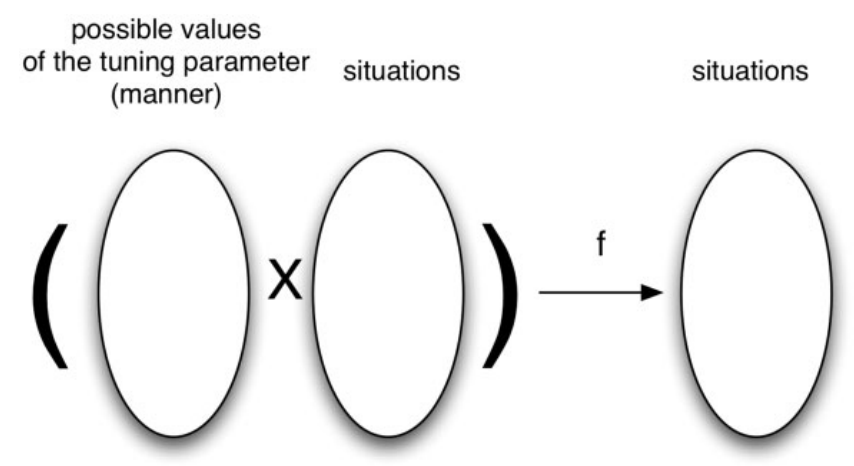

Figure 3 An action as a tunable family of similar partial function.

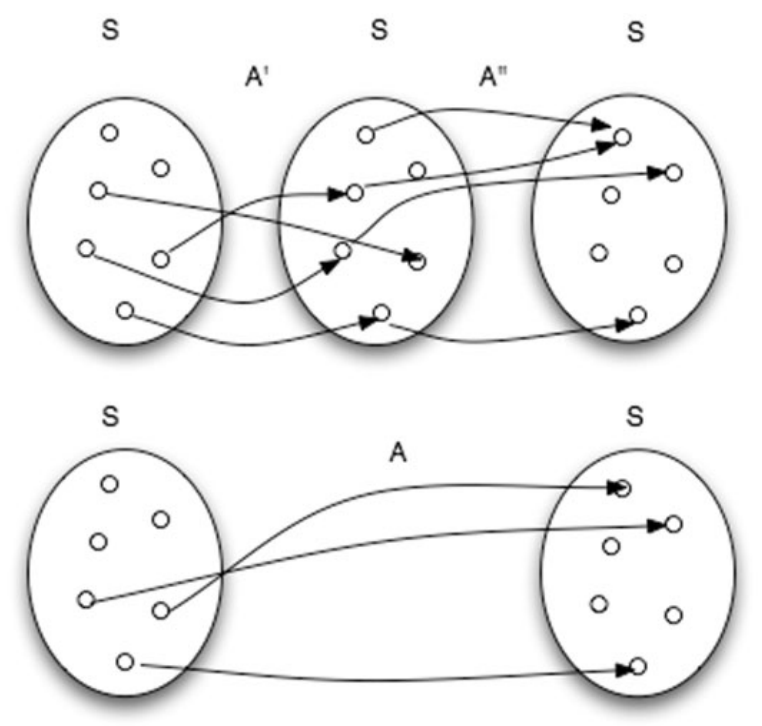

Figure 4 Composition and compilation of actions.

works. We can say that he will apply to an instance of a category of input situations (arrangements of tables in the restaurant) a composition of actions selected from the repertoire appropriate to moving tables (perhaps some sequence of pulling and pushing). As each action takes place, each component act will be characterized by the specific pattern of forces he will exert, his manner of performing. The composition of acts will lead to a sequence of transformed situations, resulting in an end, a new situation with tables more appropriately arranged, an end activated, perhaps, by his having noticed the time of day. 


\subsection{Mutually compatible actions}

The picture of performance to this point has been illustrated with abstract activities that could be either individual or collective. The elements required for the representation of multi-actor performance are already contained in the framework presented. However, it is useful to discuss more explicitly how joint action of multiple individuals is represented quite naturally within the system we have described.

First of all, our framework provides a natural way to restrict what actions can be combined: two or more actions can be combined when the range of the first is the domain of the second one and the first provides the input to the second one (e.g., preparing a subassembly for the next assembly step) or when individuals act on a common target (range) exerting a joint effect on it (e.g., jointly moving an object).

Following this notion of combined actions, we characterize mutual compatibility as follows: as long as a combination of actions satisfies agents' activated goals, such actions are mutually compatible. As a corollary, this definition implies that there may be cases in which actions fail to compose (see Figure 5), an important source of breakdown.

While this notion of mutually compatible actions may, on the surface, be suggestive of notions of equilibrium, it is in fact quite a different criterion. It implies neither optimization (it only requires some form of satisficing) nor correct beliefs about other players' strategies (e.g., an agent might ignore what other agents would do as a response to a different action of his). The notion of mutual compatibility naturally extends the idea of feasibility that Simon (1951) put forward in his work on the employment contract. However, while the notion of mutual compatibility retains a sense of intentionality, it is a weak one, weaker indeed than that of bounded rationality. Goals are activated, which means that they may result from unintentional priming. In addition, goals need not be causally related to actions (as in the example of superstitious learning below).

To make this clearer, imagine the following sequential action template, of which we will explore a few variants.

(T1) Coupled compatibility: In a given situation S, Mary has learned to activate the goal $\mathrm{X}$, and triggers action $\mathrm{Y}$ that generates situation S'. In turn, when John sees situation S' (and maybe action Y), he activates goal J, which in turn activates action $\mathrm{K}$. The outcome of Y and $\mathrm{K}$ satisfies the activated goals of both.

As the following instances of the template show, there need not be correct beliefs about other unchecked combinations of actions-indeed, there need not be such beliefs at all. What's more, actions can be compatible even when beliefs on the actual path of action are incorrect.

(T1a) Trivial compatibility: Independent actions that are mutually compatible. Mary has developed the habit to take her car at 9 am to reach the university where she is a student and thus leaves her parking place free all day long. John has developed the 


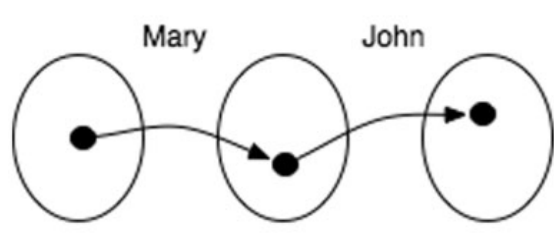

actions combine

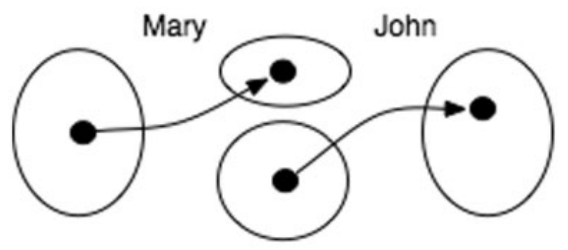

actions don't combine

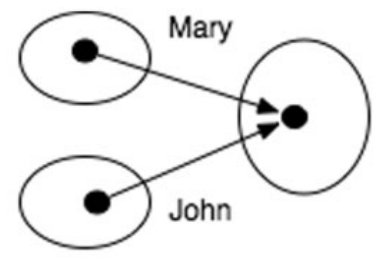

actions combine

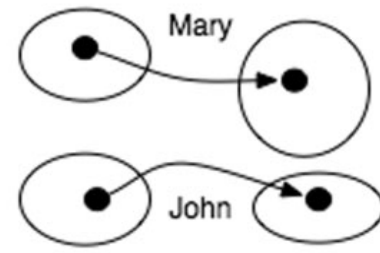

actions don't combine

Figure 5 Combination and breakdown.

habit to drive at 9 am to his work place, which is where Mary lives, and park at the place left free by Mary. John and Mary may literally ignore each other's existence, and still have compatible, mutually reinforcing habits.

(T1b) Routinized joint action: Mary performs action A which is necessary for John to complete a production task performing action B. This order of operations was established some time ago and, since it generates satisfactory performance, it has never been modified. Actually, Mary would be more satisfied and efficient performing B and John would enjoy more activity A. However, given this swapping roles alternative has never been tried, they are unaware of its superiority.

(T1c) Superstitious beliefs: Mary performs each day a superfluous act (such as bringing a cup of coffee to John at the beginning of the work day) because she thinks this will induce cooperative behavior on the part of John (this belief is a consequence of an initial event: the first day of work she brought a coffee to John and John was very kind). In fact, John displays cooperative behavior independently of the coffee (he is a cooperative type), and may actually find this behavior of Mary excessive, but out of courtesy he does not disclose his thoughts. Mary continues to believe that bringing coffee is inducing a cooperative reply. In this case, mutually compatible behaviors persist despite incorrect beliefs on the part of Mary. ${ }^{11}$

While we do not address in this article the political aspects of collective performance, we point to a few natural connections. The notion of mutually compatible

${ }^{11}$ This example is reminiscent of the well-known problems of true beliefs (Gettier, 1963). 
actions refers to a space that can be interpreted as a "zone of possible agreements" within which different negotiation solutions can be reached. This perspective is suggested by Simon's (1951) notion of an area of acceptance with regard to the employment relationship. However, it is important to stress that this space of agreements has a local nature-compatibility can hold under some circumstances and possibly in only some of the collective performance dimensions. For instance, consider Figure 6. Overall, A and B are incompatible (they don't overlap). If the two actors focus their attention on the " $\mathrm{x}$ " dimension, the overlap of A's and B's zone of acceptance projects onto a range of compatibility of $\mathrm{x}_{\mathrm{c}}$ on the $\mathrm{x}$ axis. Similarly, if attention is focused on the " $y$ " dimension, the two zones of acceptance project to a region of mutual compatibility indicated by $y_{c}$. This clearly suggests that mutual compatibility can be associated more often to a kind of truce than to a global agreement. It can hold with the help of deferral, deliberate blinders, and luck. However, it is exposed to the risk of breakdown unless, as Nelson and Winter suggested (1982), further effort is put in preserving the limited conditions under which it can remain stable.

Thus, mutual compatibility can be considered as a local property of a set of actions. Our framework allows one to differentiate at least three main forms of local compatibility. Local, but not global compatibility, may arise when (i) some, but not all, agents have mutually compatible actions; (ii) a set of actions is mutually compatible under some circumstances (a subset of the domain), but not over all circumstances; (iii) actions may be compatible under some dimensions of the domain, but not over all dimensions of it (again see Figure 6). Case (i) suggests grounds for (implicit) coalitional phenomena, while the second case (ii) suggests a system of actions prone to occasional breakdown; the third case (iii) suggests a form of "quasi-resolution" of incompatibility by acting only on some dimensions of the domain (a clear analogy with Cyert and March's (1963) quasi-resolution of conflict).

In general, once multiple agents are introduced, the domain of joint action becomes a combination of the separate features of individuals such as situation-sets representing the world from their own, possibly different points of view, differing individual ends, repertoires, and manners of action. The resulting "product space" will contain an enormous number of elements, perhaps suggesting that finding a compatible, coordinated pattern of action for several individuals might be an extremely difficult search task. Moreover, many of these elements might be in conflict. Individuals might have conflicting categorizations of situations-sets, ends, or action repertoires.

Despite this possibility of conflict, there are factors that can work to facilitate the achievement of compatible joint action. Coordinated action and the reconciliation of major differences among actors are suggested to be achieved very differently than the manner characterized in standard models of rational-or even "boundedly rational" - interaction. We lay out in broad terms some of the facilitating mechanisms below. First of all, humans - and even other animals such as monkeys—are endowed 


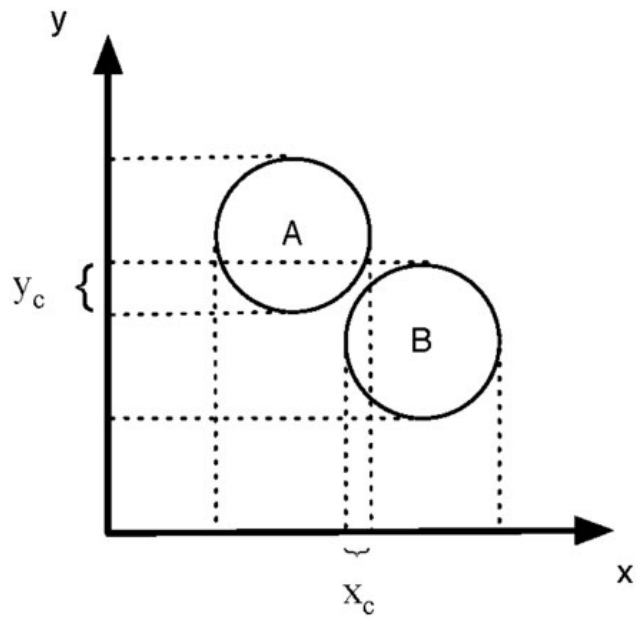

Figure 6 Overlapping zones of compatibility.

with exquisite mechanisms for recognizing the actions of others, the objects of those actions, and the feelings that go with them (Rizzolatti and Sinigaglia, 2008), thus providing common representations of action and intention. These capabilities also mean that we can simulate within ourselves the intentions of others, and have an improved ability to correctly anticipate their future actions simply as a product of actively observing them. A simple example of a model of coordination via mutual observation is presented in Model 3 in the Supplementary Materials. Some of the most powerful sources of multi-actor coordination operate via the psychological process of situational priming of actions and/or ends. This joint evocation of prior experience by a shared situation bypasses the making of isolated decisions by all participating actors, and thus reduces strategic uncertainty due to possible divergence of the actors' ends while increasing coordination. This simplifies enormously the problem of identifying a set of coordinated representations and of matching actions.

One important case of these processes can be seen in the chaining of actions across multiple individuals, where completion of a step by one actor, for example, in an assembly line, creates a situation (an "evoking step," March and Simon 1958) that powerfully activates ends in the actor who will complete the subsequent step. Narduzzo et al. (2000), for example, identify the frequent presence of "evoking steps" in trouble-fixing routines over a cell phone network. Some tests were performed even when unnecessary because they were triggered by a coordinated pattern of actions among maintenance technicians. This phenomenon also suggests that "compilation" of actions is not only an individual process but works also at the level of multiple agents.

The role of artifacts and their affordances (Gibson, 1979) in facilitating coordination fits as well this characterization of mutual coordination. First of all, the physical 
environment, and its set of visible action opportunities (leverages, surfaces, holes, handles), provides a highly available set of domains and ranges for individual action that hugely simplify the preliminary problem of making actions combine (see above), especially when agents can perceive the same objects at the same time. Think of the powerful effect of an assembly line layout, where the design of the line predetermines the structural conditions for actions. Furthermore, affordances may indeed show how some actions can be coordinated to achieve visible common goals, with the double effect of triggering specific sets of actions but also of making (compatible) goals more salient, thus potentially activating them in agents. Traffic roundabouts are a simple example of this.

"Boundary objects" (Star and Griesmer 1989) are an especially interesting class of devices favoring mutual compatibility to analyze within the framework proposed here. Star and Griesmer (1989: 393) have observed that often human cooperation has to solve the problem that multiple agents have different representations and ways to talk about a task requiring joint action. In such a context, boundary objects create a fundamental role in making interaction possible and effective: "They have different meanings in different social worlds but their structure is common enough to more than one world to make them recognizable, a means of translation. The creation and management of boundary objects is key in developing and maintaining coherence across intersecting social worlds." For example, in a new product design team a GANTT chart may act as a boundary object, as it helps different design problems to be coordinated on the time dimension (Carlile, 2002). In the language of our framework, boundary objects provide interfaces that capture projections of each agents' representations (partial sets of dimensions) and internal functions that connect actions of otherwise incompatible subjective worlds (thus providing an example of "quasi-resolution" of incompatibility). A boundary object maps different interfaces that provide projections (reduced dimensionality representations) of agents' representations and translates them via the object's internal logic.

Situation-sets are representations that may, in principle, be quite different among individuals. However, many other aspects of the world work to bring the situationsets of multiple individuals into a workable alignment. Situations-sets incorporate the presence of other persons, and these individuals are, in turn, powerful activators of ends and of possible actions. Moreover, many simple processes of cognitive change will function to create alignment of situations-sets, for example, through joint experience with prototypes and categories. (We provide a model of category alignment in the Supplementary Materials). At the level of action, in models that represent individual actors choosing in advance from large sets of discrete options, both individual repertoires and manner appear to offer many opportunities for conflict and coordination failure. However, real action unfolds over time, often with joint visibility and feedback at rates that can make mutual adjustment quite feasible. When two waiters push a table, they do not have to choose ahead of time how hard each will push. They can find a mutually consistent pattern within the first second of 
moving together. Thus, dynamic feedback may provide an important guide to selecting compatible actions (see also Simon, 1955).

\subsection{Instantiating the framework}

We have developed our framework with an eye to being sufficiently rich so as to capture the fundamental processes underlying collective performance and yet keeping the number of premises at a minimum. However, instantiating 12 distinct premises poses considerable modeling challenges. In an extensive Supplementary Materials, we provide three "live" web models that allow one to experience the dynamic nature of critical elements of collective performance, including mutual adaptation leading to compatible cognitive structures (web model S1), the interplay between the triggering of states-action patterns among individuals (web model S2), and a model (web model S3) of distributed action and cognition. Finally, we provide an illustrative application of most of the model elements in a single simple (toy) model of assembly (web model S4).

The first web model highlights the role of categorical reasoning in collective performance. Situations, even if never experienced before, are mapped to their most proximate prototype and, in turn, associated category. This mapping to categories is influenced by the salience weighting on different facets of the setting. Agents may, as a result, differ in their perceptions of a situation both as a result of their prototypes and differences in weight applied to different attributes. These differences may result in instances of coordination failure between agents. The model illustrates how the mutual adaptation of salience weights might resolves such coordination failures.

The second web model takes as its context the "transform-the-target" game introduced by Cohen and Bacdayan (1994) and examined by a number of other scholars (Egidi and Narduzzo, 1997; Wang and Zhang, 2008). This experimental context provides a powerful setting in which to see the emergence of recurring action interaction patterns among individuals. We use this context to highlight how the construct of functions can characterize action patterns. These functions take as their argument both features of the situation and activated-ends. Further, we are able to illustrate the compatibility among a set of individuals characterized in this manner or the possibility of breakdowns in the interactions in which a situation does not evoke a specific action or situation-actions pairs fail to achieve activated-ends.

The third web model involves a setting in which joint action may be useful and complementary or where it may be disruptive and interfering depending on the particular situation settings and particular actions activated. Thus, individuals face the challenge of sorting themselves into mutually compatible subgroups, which in turn requires some (implicit) partitioning of the environments that they face. This dynamic is treated as a process of Hebbian, or associative, learning (Hertz et al., 1991). Some subsets of actors receive signals from the external environment and generate actions based on their existing response repertoire and other actors respond to these acts with their own actions. We examine the emergence of stable patterns of 
behavior and illustrate how the likelihood of different behavior patterns is related to the process by which states are categorized by the actors.

The final illustrative application examines joint action in a production task and shows how associations between situation representations, activated-ends, and actions can characterize coordinated collective performance.

These models provide instantiations of the modeling premises we have articulated. While we have indicated some tentative results and properties of the models, the exercises are intended to demonstrate possible paths forward rather than the full realization of those possibilities.

\section{Conclusion}

This work has had three interrelated objectives:

1. To define a concept of collective performance that brings together a range of recurring organizational action patterns studied under labels such as "routine," "practice," "capability," or "genre." We have suggested that all these forms of organizational action share important properties in common because all involve elements of action based in habit and therefore have qualities that are not wellrepresented by models of deliberative choice.

2. To make explicit a framework of assumptions about such action patterns that reflect what observation and prior theory have shown to be the characteristic properties of collective performance. We have striven to be especially explicit and precise in presenting what we take as micro-foundational ideas underpinning theories of recurring organizational action.

3. To demonstrate that there are rich sets of well-known examples from the literature on the one hand, and of formal representations on the other, that conform to the proposed properties, suggesting that the framework has a strong potential to increase the precision, generality, and logical coherence of our theories. In both our text and in the modeling examples of the Supplementary Materials, we have been deliberately eclectic in choosing the examples, and brief in presenting them, so that more variety could be demonstrated. Our hope is that others will thereby be attracted to augment this approach.

Efforts to better understand the phenomena of collective performance have been seriously impeded, in our view, by the difficulties that confront theorists in specifying the nature of recurring organizational action and in reasoning through the implications of their assumptions. Consequently, our research literatures abound in apparent paradoxes and terminological disputes. To a large extent these difficulties are the product of squeezing the organic forms of recurring actions into the rectilinear boxes that suggest themselves when we foreground deliberate choice. We have responded by trying to specify explicitly the properties our concepts should have if we foreground instead recurring action grounded in habit as contemporary psychology and 
neuroscience now understands it: rooted in non-declarative (or "procedural") memory for action forms, in specialized systems for perceiving actions (in contrast with those for perceiving objects), in largely implicit categorizations driven by prototypes, in emotional responses to events, and activated through the interaction among these elements and with the context of action-all of these being processes to which actors have limited conscious verbal access.

We elaborated this action-emphasizing stance in the system of definitions and properties $(\mathrm{A}-\mathrm{L})$ laid out in the opening section of our article, and then presented a set of modeling examples. They demonstrate that incorporating properties that honor the habit-based character of collective performance is not necessarily at odds with increasing the precision of our concepts and inferences. The examples show that we can capture many phenomena such as old actions adapted to new contexts, actors with disparate perceptions of "the same" situation and therefore with inconsistent action dispositions, or memory for action patterns that is distributed across a group. These are examples of phenomena that are frequently reported by thoughtful organizational observers but which fit awkwardly within our theories if we picture action as flowing unproblematically from conscious choices based upon explicitly defined categories and exogenously fixed goals.

Formulization requires one to be explicit about one's assumptions and arguments. The arguments put forth here are not entirely novel, but they are couched with a degree of explicitness and a measure of coherence that has not, in our view, been typical in prior discussions of collective performance, or routines, that are anchored in a behaviorally plausible view of individual action. Formulizations are themselves typically incomplete and ours is no exception. While we point to how the idea of mutual compatibility might speak to the concept of "routines as truce," that political sensibility is not strongly developed here. Also not explicitly developed in our discussion is the role of emotion, whether as dispositional or situational attributes of actors, though clearly the affect of actors may importantly influence saliency weightings that we argue are critical in determining how different situations are encoded. Despite these limitations, it is our hope that this work, both analytical and psychologically grounded, speaks to the ongoing interest in the micro-foundations of collective performance and provides a useful foundation for such efforts.

\section{Supplementary Material}

Suppementary material, including interactive models, is available at: http://venus. unive.it/warglien/ICC/WebSuppClew0/clew0.html

\section{References}

Aarts, H. and A. Dijksterhuis (2000), 'Habits as knowledge structures: automaticity in goaldirected behavior,' Journal of Personality and Social Psychology, 78, 53-63. 
Abell, P., T. Felin and N. Foss (2008), 'Building micro-foundations for the routines, capabilities, and performance links,' Managerial and Decision Economics, 29, 489-502.

Adler, P. S. and R. Cole (1993), 'Designed for learning: a tale of two auto plants,' Sloan Management Review, 34, 85-94.

Adler, P. S., B. Goldoftas and D. I. Levine (1999), 'Flexibility versus efficiency?: a case study of model changeovers in the Toyota Production System,' Organization Science, 10, 43-68.

Argote, L. (1999), Organizational Learning: Creating, Retaining, and Transferring Knowledge. Kluwer Academic: Boston, MA.

Aristotle (1992), The Complete Works of Aristotle. InteLex Corporation: Clayton, GA.

Ashforth, B. E. and Y. Fried (1988), 'The mindlessness of organizational behaviors,' Human Relations, 41, 305-329.

Barendreght, H. P. (1984), The Lambda Calculus. Elsevier Science V: Amsterdam, The Netherlands.

Bargh, J. A. (2005), 'Bypassing the will,' in The New Unconscious. Oxford University Press: Oxford, UK \& New York, NY, pp. 37-60.

Bargh, J. A., P. M. Gollwitzer and G. Oettingen (2010), 'Motivation,' in Handbook of Social Psychology. Wiley: New York, NY.

Barrett, F. J. (1998), 'Creativity and improvisation in jazz and organizations: implications for organizational learning,' Organization Science, 9, 605-622.

Barwise, J. and J. Perry (1983), Situations and Attitudes. MIT Press: Cambridge, MA.

Berliner, P. F. (1994), Thinking in Jazz. University of Chicago Press: Chicago, IL.

Birnholtz, J., M. D. Cohen and S. V. Hoch (2007), 'Organizational character: on the regeneration of Camp Poplar Grove,' Organization Science, 18, 315-332.

Bourdieu, P. (1990), The Logic of Practice. Polity Press: Cambridge, UK \& Basil Blackwell: Oxford, UK.

Carlile, P. R. (2002), 'A pragmatic view of knowledge and boundaries: boundary objects in new product development,' Organization Science, 13, 442-455.

Chassang, S. (2010), 'Building routines: learning, cooperation, and the dynamics of incomplete relational contracts,' American Economic Review, 100, 448-465.

Cohen, M. D. (2007), 'Reading Dewey: reflections on the study of routine,' Organization Studies, 28, 773-786.

Cohen, M. D. (2012), 'Perceiving and remembering routine action: fundamental micro-level origins,' Journal of Management Studies, 49, 1383-1388.

Cohen, M. D. and P. Bacdayan (1994), 'Organizational routines are stored as procedural memory: evidence from a laboratory study,' Organization Science, 5, 554-568.

Cohen, M. D., R. Burkhart, M. Egidi, L. Marengo, M. Warglien and S. Winter (1996), 'Contemporary issues in research on routines and other recurring action patterns of organization,' Industrial and Corporate Change, 5, 663-698. 
Cusumano, M. A. (1985), The Japanese Automobile Industry: Technology and Management at Nissan and Toyota. Harvard University Press: Cambridge University Press.

Cyert, R. M. and J. G. March (1963), A Behavioral Theory of the Firm. Prentice-Hall: Englewood Cliffs, NJ.

Davey, B. A. and H. A. Priestley (2002), Introduction to Lattices and Order. Cambridge University Press: Cambridge, UK.

Decety, J. and J. A. Sommerville (2003), 'Shared representations between self and other: a social cognitive neuroscience view,' Trends in Cognitive Sciences, 7, 527-533.

Dewey, J. (1922), Human Nature and Conduct: An Introduction to Social Psychology. H. Holt and Company: New York, NY.

Dosi, G., R. Nelson and S. G. Winter (eds) (2000), Nature and Dynamics of Organizational Capabilities. Oxford University Press: Oxford, UK.

D'Adderio, L. (2011), 'Artifacts at the centre of routines: Performing the material turn in routines theory,' Journal of Institutional Economics, 7, 197-230.

Egidi, M. and A. Narduzzo (1997). 'The emergence of path-dependent behaviors in cooperative contexts,' International Journal of Industrial Organization, 15: 677-709.

Feldman, M. S. (2000), 'Organization routines as a source of continuous change,' Organization Science, 11, 611-629.

Feldman, M. S. and B. T. Pentland (2003), 'Reconceptualizing organizational routines as a source of flexibility and change,' Administrative Science Quarterly, 48, 94-121.

Felin, T. and N. J. Foss (2005), 'Strategic organization: a field in search of micro-foundations,' Strategic Organization, 3, 441-455.

Felin, T. and N. J. Foss (2009), 'Organizational routines and capabilities: historical drift and a course-correction toward microfoundations,' Scandinavian Journal of Management, 25, $157-167$.

Gettier, E. (1963), 'Is justified true belief knowledge?' Analysis, 23, 121-123.

Gibson, J. J. (1979), The Ecological Approach to Visual Perception. Houghton Mifflin: Boston, MA.

Giddens, A. (1984), The Constitution of Society: Outline of the Theory of Structuration. University of California Press: Berkeley, CA.

Graybiel, A. M. (2008), 'Habits, rituals, and the evaluative brain,' Annual Review of Neuroscience, 31, 359-387.

Gärdenfors, P. (2000), Conceptual Spaces: The Geometry of Thought. The MIT Press: Cambridge, MA.

Hertz, J., A. Krogh, and R. Palmer (1991), Introduction to the theory of neural computation. Addison Wesley: Reading, MA.

Howard-Grenville, J. A. (2005), 'The persistence of flexible organizational routines: the role of agency and organizational context,' Organization Science, 16, 618-636.

James, W. (1981), The Principles of Psychology. Harvard University Press: Cambridge, MA. 
Jeannerod, M. (1997), The Cognitive Neuroscience of Action. Blackwell: London, UK.

Kane, A. A., L. Argote and J. M. Levine (2005), 'Knowledge transfer between groups via personnel rotation: effects of social identity and knowledge quality,' Organizational Behavior and Human Decision Processes, 96, 56-71.

Lawvere, F. W. and S. H. Schanuel (1991), Conceptual Mathematics: A First Introduction to Categories. Buffalo Workshop Press: Buffalo, NY.

Levin, B. and M. Rappaport Hovav (1991), 'Wiping the slate clean: A lexical semantic exploration,' Cognition, 41, 123-151.

Levinthal, D. and C. Rerup (2006), 'Crossing an apparent chasm: bridging mindful and lessmindful perspectives on organizational learning,' Organization Science, 17, 502-513.

Liker, J. K. (2003), The Toyota Way Fourteen Management Principles from the World's Greatest Manufacturer. McGraw-Hill: New York, NY.

MacDuffie, J. P. (1997). 'The road to 'root cause': Shop-floor problem-solving at three auto assembly plants,' Management Science, 43, 472-502.

March, J.G. and H. A. Simon (1958). Organizations. New York: Wiley.

Massen, C. and W. Prinz (2009), 'Movements, actions and tool-use actions: an ideomotor approach to imitation,' Philosophical Transactions of the Royal Society B: Biological Sciences, 364, 2349-2358.

Morewedge, C. K. and D. Kahneman (2010), 'Associative processes in intuitive judgment,' Trends in Cognitive Sciences, 14, 435-440.

Mullainathan, S., J. Schwartzstein and A. Shleifer (2008), 'Coarse thinking and persuasion,' Quarterly Journal of Economics, 123, 577-619.

Narduzzo, A., E. Rocco and M. Warglien (2000), 'Talking about routines in the field: the emergence of organizational capabilities in a new cellular phone network company,' in G. Dosi, R. Nelson and S. Winter (eds), The Nature and Dynamics of Organizational Capabilities. Oxford University Press: Oxford, UK.

Neal, D. T., W. Wood, J. S. Labrecque and P. Lally (2012), 'How do habits guide behavior?: perceived and actual triggers of habits in daily life,' Journal of Experimental Social Psychology, 48, 492-498.

Nelson, R. and S. Winter (1982), An Evolutionary Theory of Economic Change. Belknap Press of Harvard University: Cambridge, MA.

Norman, J. (2002), 'Two visual systems and two theories of perception: An attempt to reconcile the constructivist and ecological approaches,' Behavioral and Brain Sciences, 25, 73-144.

Orlikowski, W. J. (2002), 'Knowing in practice: Enacting a collective capability in distributed organizing,' Organization Science, 13, 249-273.

Pentland, B. T. and M. S. Feldman (2005), 'Organizational routines as a unit of analysis,' Industrial and Corporate Change, 14, 793-815.

Pentland, B. T., T. Haerem and D. Hillison (2010), 'Comparing organizational routines as recurrent patterns of action,' Organization Studies, 31, 917-940. 
Pentland, B. T. and H. H. Rueter (1994), 'Organizational routines as grammars of action,' Administrative Science Quarterly, 39, 484-510.

Ping, R. M., S. Dhillon and S. L. Beilock (2009), 'Reach for what you like: the body's role in shaping preferences,' Emotion Review, 1, 140-150.

Polanyi, M. (1958), Personal Knowledge: Towards a Post-Critical Philosophy. University of Chicago Press: Chicago, IL.

Rico, R., M. Sánchez-Manzanares, F. Gil and C. Gibson (2008), 'Team implicit coordination processes: a team knowledge-based approach,' The Academy of Management Review, 33, $163-184$.

Ricoeur, P. (1975), La Métaphore Vive. Le Seuil: Paris, France.

Rizzolatti, G. and C. Sinigaglia (2008), Mirrors in the Brain: How Our Minds Share Actions and Emotions. Oxford University Press: Oxford, UK.

Rosch, E. and C. B. Mervis (1975), 'Family resemblances: Studies in the internal structure of categories,' Cognitive Psychology, 7, 573-605.

Rumelhart, D. E. (1992), 'Toward a microstructural account of human reasoning,' in Connectionism: theory and practice. Oxford University Press: New York, NY, pp. 69-83.

Savage, L. J. (1954), The Foundations of Statistics. Wiley: New York, NY.

Sebanz, N., H. Bekkering and G. Knoblich (2006), 'Joint action: bodies and minds moving together,' Trends in Cognitive Sciences, 10, 70-76.

Seger, C. A. and B. J. Spiering (2011), 'A critical review of habit learning and the Basal Ganglia,' Frontiers in Systems Neuroscience, 5, 66.

Seiden, S. C. and P. Barach (2006), 'Wrong-side/wrong-site, wrong-procedure, and wrongpatient adverse events: Are they preventable?' Archives of Surgery, 141, 931-939.

Simon, H. (1951), 'A formal theory of the employment relationship,' Econometrica, 3, 293-305.

Simon, H. (1955), 'A behavioral model of rational choice,' Quarterly Journal of Economics, 69, 99-118.

Singley, M. K. and J. R. Anderson (1989), The Transfer of Cognitive Skill. Harvard University Press: Cambridge, MA.

Squire, L. and E. R. Kandel (1999), Memory: From Mind to Molecules. Scientific American Library: New York, NY.

Star, S. L. and J. R. Griesemer (1989), 'Institutional ecology, "translations" and boundary objects: Amateurs and professionals in Berkeley's Museum of Vertebrate Zoology, 1907-39,' Social Studies of Science, 19, 387-420.

Teece, D. J., G. Pisano and A. Shuen (1997), 'Dynamic capabilities and strategic management,' Strategic Management Journal, 18, 509-533.

Wang, J., and G. Zhang (2008) 'Knowledge, Routines and Performance in Collective Problem Solving,' Acta Psychologia Sinica, 40, 862-872. 
Warglien, M. and P. Gärdenfors (2013), 'Semantics, conceptual spaces and the meeting of minds,' Synthese, 190, 2165-2193.

Warglien, M., P. Gärdenfors, and M. Westera (2012), 'Event structure, conceptual spaces and the semantics of verbs,' Theoretical Linguistics, 38, 159-193.

Weber, K. (2005), 'A toolkit for analyzing corporate cultural toolkits,' Poetics, 33, 227-252.

Weick, K. (1995), Sensemaking in Organizations. Sage Publications: Thousand Oaks, CA.

Weick, K. E. (1990), 'The vulnerable system: an analysis of the Tenerife air disaster,' Journal of Management, 16, 571-593.

Weick, K. E. (1998), 'Improvisation as a metaphor for organizing,' Organization Science, 9, 543-555.

Weick, K. E. and K. H. Roberts (1993), 'Collective mind in organizations: heedful interrelating on flight decks,' Administrative Science Quarterly, 38, 357-381.

Weick, K. E. and K. M. Sutcliffe (2006), 'Mindfulness and the quality of organizational attention,' Organization Science, 17, 514-524.

Weick, K. E., K. M. Sutcliffe and D. Obstfeld (2005), 'Organizing and the process of sensemaking,' Organization Science, 16, 409-421.

Winter, S. G. (1994), 'Organizing for continuous improvement: Evolutionary theory meets the quality revolution,' in J. Baum and J. Singh (eds), Evolutionary Dynamics of Organizations. Oxford University Press: Oxford, UK and New York, NY, pp. 90-108.

Womack, J. P., D. T. Jones and D. Roos (1990), The Machine That Changed the World. Rawson Associates, MacMillian Publishing Company: New York, NY.

Wood, W. and D. T. Neal (2007), 'A new look at habits and the habit-goal interface,' Psychological Review, 114, 843-863.

Yates, J. and W. Orlikowski (2002), 'Genre systems: Structuring interaction through communicative norms,' Journal of Business Communication, 39, 13-35. 